\title{
Diseño y validación de una escala para medir la gestión de la identidad digital
}

Design and Validation of a Scale to Measure Digital Identity Management

Projeto e validação de uma escala para medir o gerenciamento de identidade digital

Gustavo Antonio Huerta Patraca Universidad Veracruzana, Facultad de Pedagogía, México

gushuerta@uv.mx https://orcid.org/0000-0001-5168-974X

Carlos Arturo Torres Gastelú Universidad Veracruzana, México ctorres@uv.mx https://orcid.org/0000-0003-2527-9602

Agustín Lagunes Domínguez Universidad Veracruzana, México aglagunes@uv.mx https://orcid.org/0000-0002-8101-154X 


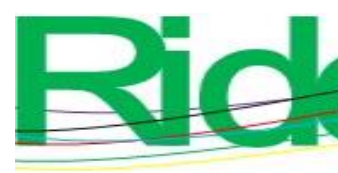

Revista Iberoamericana para la Investigación y el Desarrollo Educativo ISSN 2007-7467

Olkin (KMO) index of 0.728 were obtained globally for the five subscales. The final version contains 32 items grouped into five factors. The IDentifica2.0 scale has an acceptable level of reliability and validity to measure the management of digital identity, contributing to the analysis of this digital competence

Keywords: reliability, perception scale, university student, digital identity, measurement instrument, measurement, validity.

\section{Resumo}

A gestão da identidade digital implica um processo permanente e consciente de tratamento da informação pessoal e dos conteúdos partilhados na Internet, com vista à protecção da privacidade e da reputação digital. O objetivo do artigo é descrever o processo de desenho e validação da escala de percepção intitulada IDentifica2.0, criada para medir a gestão da identidade digital, instrumento utilizado para pesquisa de doutorado em Sistemas e Ambientes Educacionais da Universidade Veracruzana, México. A escala Likert desenvolvida contém cinco dimensões: criação da identidade digital, visibilidade, privacidade e segurança na Internet, reputação digital e efeitos da identidade digital na esfera pessoal. Foi aplicado a 319 alunos da licenciatura em Pedagogia da Universidade Veracruzana ( $88 \%$ mulheres e 12\% mulheres). Uma análise fatorial exploratória (AFE) e análise fatorial confirmatória (CFA) foram realizadas para determinar sua validade, o que levou à eliminação de itens com baixa ou nenhuma correlação. Como parte dos resultados, um alfa de 0,80 e um índice Kaiser-Meyer-Olkin (KMO) de 0,728 foram obtidos globalmente para as cinco subescalas. A versão final contém 32 itens agrupados em cinco fatores. A escala IDentifica2.0 tem um nível aceitável de confiabilidade e validade para medir a gestão da identidade digital e apóia a análise dessa competência digital.

Palavras-chave: confiabilidade, escala de percepção, estudante universitário, identidade digital, instrumento de medida, medida, validade.

Fecha Recepción: Julio 2020

Fecha Aceptación: Mayo 2021 


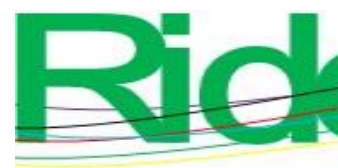
Revista Iberoamericana para la
Investigación y el Desarrollo Educativo
ISSN $2007-7467$

\section{Introducción}

Castañeda y Camacho (2012) consideran que la identidad digital se conforma a partir de la información personal que se comparte en Internet, además de la interacción social generada con otros usuarios. Para Peachey y Childs (2011), la identidad digital "es proyectada por lo que una persona hace en la Red: qué dice, cómo lo dice, el lenguaje que usa, sus temas de interés y un largo etcétera que configuramos básicamente cada vez que hacemos click en el ordenador” (p. 7). Por lo que se puede considerar como el conjunto de datos personales que se publican en Internet a través de los cuales se autodenomina una persona; se complementa con la información que otros usuarios intercambian y comparten con dicha persona, y se asocia a un perfil digital (Aced, Arqués, Benítez, Llodrà y Sanagustín, 2009; Ainsa, 2016; Lara, 2009; Tusa, 2018).

Prácticamente todo lo que se hace en Internet, cualquier consulta, envío de mensajes, creación de contenido o compartir información, ahonda la huella digital de cada usuario, un rastro que dice mucho sobre su comportamiento en la Red. Esta identidad digital "se construye a través de la actividad en Internet, cuando aporta textos, imágenes y vídeos a Internet, participando, en definitiva, del mundo web" (Giones y Serrat, 2010, p. 3). Implica un proceso permanente de ensayo y error (Gamero, 2009). Y está ligado a las acciones de los usuarios en los entornos digitales, a los avatares y perfiles en línea, a la información que se comparte en redes sociales, a los datos derivados de nuestros objetos digitales personales, etc. (Gabrielidis, 2015).

Para ubicar la gestión de la identidad digital en el contexto de la competencia digital, Ferrari (2013) señala, derivado del proyecto DigComp, que la competencia digital se integra por cinco áreas, que en su conjunto derivan en 21 competencias (ver tabla 1), entre ellas la competencia 2.6: gestión de la identidad digital. 


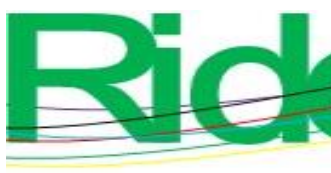

Revista Iberoamericana para la Investigación y el Desarrollo Educativo ISSN 2007 - 7467

Tabla 1. Áreas de competencias y competencias digitales

\begin{tabular}{|c|c|}
\hline Área & Competencia \\
\hline \multirow[t]{3}{*}{ 1) Información } & 1.1) Navegación, búsqueda y filtrado de información \\
\hline & 1.2) Evaluación de la información \\
\hline & 1.3) Almacenamiento y recuperación de la información \\
\hline \multirow[t]{6}{*}{ 2) Comunicación } & 2.1) Interacción mediante nuevas tecnologías \\
\hline & 2.2) Compartir información y contenidos \\
\hline & 2.3) Participación ciudadana en línea \\
\hline & 2.4) Colaboración mediante canales digitales \\
\hline & 2.5) Netiqueta \\
\hline & 2.6) Gestión de la identidad digital \\
\hline \multirow{4}{*}{$\begin{array}{l}\text { 3) Creación de } \\
\text { contenido }\end{array}$} & 3.1) Desarrollo de contenidos \\
\hline & 3.2) Integración y reestructuración \\
\hline & 3.3) Derechos de autor y licencias \\
\hline & 3.4) Programación \\
\hline \multirow[t]{4}{*}{ 4) Seguridad } & 4.1) Protección de dispositivos \\
\hline & 4.2) Protección de datos personales \\
\hline & 4.3) Protección de la salud \\
\hline & 4.4) Protección del entorno \\
\hline \multirow{4}{*}{$\begin{array}{l}\text { 5) Resolución de } \\
\text { problemas }\end{array}$} & 5.1) Resolución de problemas técnicos \\
\hline & 5.2) Identificación de necesidades y respuestas tecnológicas \\
\hline & 5.3) Innovar y utilizar la tecnología de forma creativa \\
\hline & 5.4) Identificación de lagunas en la competencia digital \\
\hline
\end{tabular}

Fuente: Ferrari (2013, p. 11)

Ahora bien, Giones y Serrat (2010) establecen que "la gestión de la identidad digital es el manejo consciente del entorno web, las oportunidades y peligros de la Red a la hora de publicar la propia información personal en la sociedad del conocimiento" (p. 2). Por su parte, para Ferrari (2013) "la gestión de la identidad digital implica crear, adaptar y gestionar una o varias identidades digitales, ser capaz de proteger su reputación digital, gestionar los datos que uno produce a través de varias cuentas y aplicaciones" (p. 11). De modo inicial, la gestión de la identidad digital 


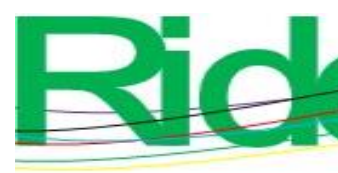

Revista Iberoamericana para la Investigación y el Desarrollo Educativo ISSN 2007-7467

contempla tres dimensiones: visibilidad, reputación y privacidad (Giones y Serrat, 2010; Pérez, 2012).

La gestión de la identidad digital se inicia desde la construcción de los perfiles de usuario (Georges, 2011; Tusa, 2018), toda vez que en Internet las personas pueden crear una identidad digital que coincida total o parcialmente con la identidad personal (Aguilar y Said, 2010; Dans, 2015; Perea, 2010), además de que se puede generar más de una identidad en el ámbito virtual (Bozkurt y Tu, 2016; Ferrari, 2013; Tusa, 2018). En suma, posee una parte personal y otra social (Castañeda y Camacho, 2012), y es autoconstruida por el usuario o conformada por las personas con las que interactúa en el ámbito virtual.

Asimismo, esta interacción que se suscita en el ámbito digital tiene una repercusión en el ámbito personal, toda vez que las acciones derivadas de la interacción virtual pueden afectar las relaciones físicas o sociales entre personas que conviven cotidianamente (Ahlquist, 2016; Fernández, 2014; Portillo, 2016). Por otro lado, se identifica también el efecto que el ámbito personal genera en los usuarios de Internet (Adriaanse y Rensleigh, 2017; Ainsa, 2016; Valderrama, 2017; Vargas, 2016). Partiendo de lo anterior, se conciben como parte de la gestión de la identidad digital la creación de los perfiles digitales, además de los efectos que las acciones del entorno virtual generan en el ámbito personal de los usuarios, por lo que la gestión de este tipo quedaría conformada por cinco dimensiones: creación de la identidad digital, visibilidad, privacidad y seguridad en Internet, reputación digital y los efectos de la identidad digital en el ámbito personal.

Se partió de la pregunta: ¿qué nivel de confiabilidad y validez posee la escala IDentifica2.0 para medir la gestión de la identidad digital en estudiantes universitarios? El objetivo de la investigación fue describir el proceso de diseño y validación de la escala IDentifica2.0. Se parte de la hipótesis de que dicho instrumento reúne los criterios de confiabilidad y validez necesarios para ser utilizada en estudiantes universitarios. 


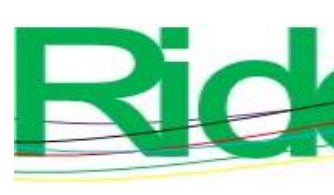

Revista Iberoamericana para la Investigación y el Desarrollo Educativo ISSN 2007-7467

\section{Estudios relacionados}

Partiendo de estas cinco dimensiones señaladas, se realizó la búsqueda de estudios similares a fin identificar las tendencias al respecto. En una primera búsqueda se encontraron 94 estudios relacionados. Tras la revisión a fondo, en apego a los criterios de congruencia y utilidad para el diseño de la escala, se trabajó con 48 estudios. Destaca que se encontraron 14 estudios de la dimensión de privacidad y seguridad en Internet, seguida de reputación digital con 12, visibilidad con nueve, efectos de la identidad personal en el ámbito personal con siete y, finalmente, seis de la dimensión de creación de la identidad digital.

Cabe precisar que algunos estudios hacen referencia a más de una dimensión (Ainsa, 2016; Kim y Choi, 2018; Mannerström, Hietajärvi, Muotka y Salmela, 2018;). Por ejemplo, Pérez (2012) y Giones y Serrat (2010) coinciden en abordar las dimensiones de visibilidad, privacidad y seguridad en Internet y reputación digital, mientras que el estudio de Castañeda y Camacho (2012) se enfoca en la creación de la identidad digital y la reputación digital. Estos estudios tienen en común el enfoque cuantitativo y que fueron realizados a través de la aplicación de escalas de percepción o encuestas.

La creación de la identidad digital implica un proceso cuyo punto de partida es la identidad personal (Mannerström et al., 2018; Ainsa, 2016; Bjerede, 2015). Es decir, se conjuga lo que las personas son desde un punto de vista biológico, social y psicológico con la forma en que se identifican como usuarios de Internet: puede existir correspondencia directa o dar lugar a diferencias entre uno y otro polo (Kim y Choi, 2018; Wise y O’Byrne, 2015). Asimismo, no hay que perder de vista un punto arriba ya mencionado: los usuarios pueden generar más de una identidad en un mismo servicio de Internet, vinculado a varios perfiles digitales (Castañeda y Camacho, 2012; Mannerström et al., 2018; Wise y O’Byrne, 2015).

En relación con la visibilidad, para Van y Steinfield (2018), así como para Pérez (2012), el proceso de interacción tecnológica entre el usuario y la información (interacción tecnológica) y el intercambio de contenido con otros usuarios (interacción social) es lo que genera la huella digital asociada a cada perfil de usuario (Beck, 2015; Galera, Hurtado y Fernández, 2014; Mannerström et al., 2018). Por supuesto, los usuarios buscan incrementar su nivel de visibilidad, que se encuentra relacionado con el alcance que logran sus publicaciones. Como es sabido, todo este contenido publicado se asocia con la ciudadanía digital (Kim y Choi, 2018; So, Wu, Xiong y King, 2018), de modo que las personas que utilizan redes sociales y demás servicios de Internet deben de cuidar la 
información que comparten (Fernández, 2014; Giones y Serrat, 2010; Kim y Choi, 2018; Pérez, 2012).

El binomio privacidad y seguridad en el mundo del Internet refiere, por un lado, a implementar acciones en los dispositivos para proteger la información que se considera valiosa (Castillejos, Torres y Lagunes, 2016; Giones y Serrat, 2010; Pérez, 2012; Rodríguez y Magdalena, 2016). Y por el otro, implica también cuidarse de los distintos riesgos existentes al utilizar Internet (García, del Olmo y del Hoyo, 2017; Ortega, del Rey y Casas, 2016; de Frutos y Vázquez, 2014). Entre estos riesgos se ubican el ciberbullying o acoso en medios virtuales (Herrera, Casas, Romera, Ortega y del Rey, 2017; Sabater y López, 2015; D’Antona, Kevorkian y Russom, 2010), pishing o robo de datos con fines comerciales (Castillejos et al., 2016; Meraz, 2018), sexting o intercambio de mensajes con contenido erótico (Herrera et al., 2017) y el grooming, cuando los menores de edad están expuestos a tener contacto con personas desconocidas que pretendan hacerle algún daño (D’Antona et al., 2010). Parte de la seguridad también es el cuidado de la salud visual y postura al utilizar dispositivos por tiempo prolongado (Saiz, Ronco y Echegaray, 2017).

Hay estudios que analizan la percepción de estos riesgos por parte de los jóvenes. Algunos de estos participantes jóvenes no se visualizan vulnerables ante estas situaciones, y reconocen además tener ciertos cuidados al navegar por Internet (Herrera et al., 2017; Moreno, Gajardo y Parra, 2016; Ramos, López y Torrecillas, 2018); otros, sin embargo, confiesan que han sufrido alguno de los actos arriba mencionados, lo que los ha llevado a fortalecer las medidas de seguridad y privacidad de sus perfiles digitales (de Frutos y Vázquez, 2014; Rodríguez y Magdalena, 2016; Sabater y López, 2015).

Como ya se ha mencionado, la reputación digital se construye a partir del contenido que cada usuario genera y comparte en Internet, así como lo que otros usuarios opinan de la persona o institución (Giones y Serrat, 2010; Harrell y Lim, 2017; Pérez, 2012), por lo que se han implementado instrumentos para valorar este indicador (Dutot y Castellano, 2015; Nicholas, 2017; Núñez y Manolakis, 2016; Seker y Eryarsoy, 2015), al igual que estudios para conocer las prácticas que implementan los usuarios para generar su reputación digital y el proceso para el cuidado de esta (Baladán y Hernández, 2016; Castañeda y Camacho, 2012; Kelly, Christen y Snyder, 2013; Telci y Kantur, 2014).

En cuanto a los efectos de la identidad digital en el ámbito personal, se ubicaron estudios que plantean dos direcciones. Por un lado, los que analizan el impacto que tiene la interacción y el contenido que han publicado en Internet en lo personal, social o laboral y que de alguna u otra 


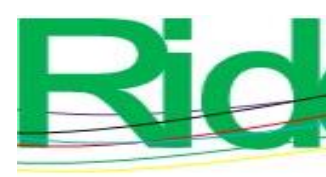
Revista Iberoamericana para la
Investigación y el Desarrollo Educativo
ISSN $2007-7467$

manera puede ayudarles o perjudicarles en cierto momento (Ahlquist, 2016; Ainsa, 2016; Baladán y Hernández, 2016; Davis, 2013); aquí también se inspecciona la influencia que cierta información vista en la Red puede ejercer sobre el aspecto ideológico y de conducta (Dalton y Crosby, 2013; Davis, 2013). Por el otro lado, están los que analizan la causa e influencia que tienen las interacciones que realiza el usuario con otras personas en un ámbito sociopersonal y la manera en que estas son expresadas en el ámbito virtual (de Frutos y Valle, 2014; Vargas, 2016).

\section{Metodología}

Para el diseño del instrumento, se realizó primeramente una revisión teórica que derivó en el establecimiento de dimensiones que permitieron la construcción de indicadores e ítems para integrar la primera versión de la escala. Esta fue revisada por expertos, se realizó el pilotaje y el análisis estadístico de datos respectivo para determinar su confiabilidad e identificación de factores a partir de un análisis factorial exploratorio (AFE) y un análisis factorial confirmatorio (AFC).

\section{Participantes}

Se eligieron de forma no probabilística a estudiantes de la licenciatura en Pedagogía de la Universidad Veracruzana, del estado de Veracruz, México. El criterio inicial de inclusión fue que estuvieran inscritos en dicho programa académico, sin distinción de edad o ciclo escolar, y que contaran con disponibilidad para participar en el estudio. Posteriormente, mediante un muestreo probabilístico simple sin reemplazo $(p=0.5, q=95 \%$, error $=5 \%)$, se seleccionó a 319 estudiantes, de los cuales $88 \%$ eran mujeres y $22 \%$ hombres.

\section{Instrumento}

Con base en la revisión de la literatura (Bozkurt y Tu, 2016; Castañeda y Camacho, 2012; Kim y Choi, 2018; Mannerström et al., 2018; Pérez, 2012; Tusa, 2017; Valderrama, 2018), se desarrolló una escala de percepción denominada IDentifica2.0 para medir la gestión de la identidad digital, entendida como el manejo consciente del entorno web, las oportunidades y peligros de la Red a la hora de publicar la propia información personal en la sociedad del conocimiento (Giones y Serrat, 2010). Este instrumento se empleó en la tesis doctoral de Sistemas y Ambientes Educativos de la Universidad Veracruzana, México. La versión piloteada está integrada por cinco 


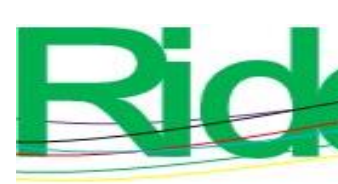

Revista Iberoamericana para la Investigación y el Desarrollo Educativo ISSN 2007 - 7467

subescalas, con 13 ítems cada una, para un total de 65 ítems, agrupados en cinco dimensiones y 15 indicadores.

Las cinco dimensiones e indicadores son:

1) Creación de la identidad digital (CID): comprende los indicadores autoidentidad y multiplicidad de identidades;

2) Visibilidad (VIS): implica huella digital e interacción sociodigital;

3) Privacidad y seguridad en Internet (PYS): integra medidas preventivas y correctivas y protección de riesgos;

4) Reputación digital (RED): abarca autopercepción y percepción social, y

5) Efectos de la identidad digital en el ámbito personal (EDID): implica los efectos de lo real a lo virtual y de lo virtual a lo real.

Algunos ítems de ejemplo por cada dimensión son los siguientes: a) Construcción de la identidad digital (13 ítems, p. ej.: "Internet me brinda un espacio para expresarme tal y como soy”), b) Visibilidad (13 ítems, p. ej.: "Interactúo con miembros de mi familia por Internet”), c) Privacidad y seguridad en Internet (13 ítems, p. ej.: "Utilizo solamente software original en mis dispositivos”), d) Reputación digital (13 ítems, p. ej.: “Considero que utilizo Internet respetando a los demás usuarios de la Red”), y e) Efectos de la identidad digital en la identidad personal (13 ítems, p. ej.: "Lo que leo y comparto en Internet ha modificado mis creencias, ideas y valores").

El instrumento se compone de tres partes. En la primera, de datos generales, se solicita información sociodemográfica y académica como edad, sexo, nombre de la escuela y ciclo escolar que cursa; en la segunda parte se presentan tres preguntas, en las que los participantes pueden marcar más de una opción, sobre los servicios de Internet que usan (correo electrónico, apps para celular, música, videos, videojuegos online, trámites de gobierno, trámites escolares y compra/venta), redes sociales utilizadas (Facebook, Whatsapp, Instagram, YouTube, Snapchap y otra app, opción en la que deben especificar), y los dispositivos que emplean para conectarse a Internet (teléfono celular, computadora de escritorio, computadora portátil, tablet, televisión/smart TV y consola de videojuegos), y en la tercera parte se incluyen los ítems con sus opciones de respuesta, cuyo formato utilizado fue de respuesta tipo Likert: Totalmente de acuerdo (TA), De acuerdo (DA), Neutral (N), En desacuerdo (ED) y Totalmente en desacuerdo (TD). Las respuestas fueron valoradas con puntajes de cuatro a uno, respectivamente. 


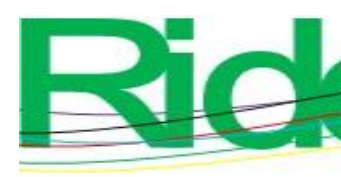

Revista Iberoamericana para la
Investigación y el Desarrollo Educativo ISSN 2007-7467

\section{Procedimiento}

Retomando la información teórica, se elaboró una primera versión del instrumento que constó de 15 ítems (cinco por cada mecanismo). Esta primera versión se sometió a pruebas de validez de contenido (teórico, cultural y lingüístico) por medio de un juicio de expertos (tres investigadores en la temática y dos docentes universitarios del área de tecnologías de la información y la comunicación [TIC] y educación). Tras sus observaciones, se consideró mantener únicamente los 65 ítems que obtuvieron un índice Kappa de concordancia entre jueces $\geq 0.80$; cuatro de ellos redundan en sus opiniones a favor respecto del contenido y coherencia interna del instrumento, mientras que el otro lector señaló algunas observaciones que ayudaron a mejorar su estructura y redacción.

Para poder llevar a cabo el pilotaje del cuestionario previo al análisis de las propiedades psicométricas, se presentó el proyecto de investigación a la dirección y secretaría académica de la Facultad de Pedagogía de la Universidad Veracruzana. Después de ello, se invitó a participar a los estudiantes, haciendo de su conocimiento los objetivos del estudio y solicitando su participación voluntaria. La duración de la aplicación fue, en promedio, de 20 minutos.

$\mathrm{Al}$ analizar las propiedades psicométricas del instrumento se establecieron evidencias de confiabilidad, determinada a partir de la consistencia interna de los puntajes obtenidos por el alfa de Cronbach, y validez de constructo, establecida mediante los análisis factoriales exploratorio y confirmatorio.

\section{Análisis de datos}

En primer lugar, se calcularon las medias, desviaciones estándar, asimetría y curtosis de los ítems como indicadores de normalidad univariada, así como también se efectuó un análisis de consistencia interna de cada escala mediante el alfa de Cronbach. Posteriormente, se realizó el AFE deductivo, a partir de un método de extracción por componentes principales y rotación varimax, empleando el software estadístico SPSS en su versión 23. Finalmente, se llevó a cabo el AFC con el software AMOS versión 23, utilizando el método de estimación de máxima verosimilitud (ML). 


\section{Resultados}

\section{Análisis descriptivos}

Se realizaron análisis descriptivos como evidencia de la normalidad univariada en la distribución de los puntajes de los ítems. En la tabla 2 se muestran los valores de medias (M), desviaciones estándar (DE), asimetría y curtosis de todos los ítems que componen el cuestionario IDentifica2.0. Los valores de asimetría y curtosis sugieren la existencia de normalidad univariada en la distribución de los puntajes de los ítems, debido a que los valores de asimetría y curtosis se consideran aceptables, al encontrarse en los rangos de -3 a +3 y de -10 a +10 , respectivamente (Griffin y Steinbrecher, 2013; Kline, 2016).

Por consiguiente, todos los ítems muestran normalidad univariada en sus puntajes, con excepción de los ítems VIS14, VIS20, PYS36, PYS37 Y PYS38, los cuales presentan valores de curtosis por encima de +3 . Sin embargo, en pruebas de modelaje de ecuaciones estructurales como el AFC se admiten valores curtosis de hasta +10 debido a la robustez de la prueba (Griffin y Steinbrecher, 2013; Kline, 2016).

Por otro lado, el ítem VIS14 fue el que presentó menor variabilidad en sus opciones de respuesta (alrededor de las opciones tres y cuatro), al tener la menor desviación estándar (0.69), con media de 3.63. Por su parte, el ítem CID9 fue el de mayor variabilidad (alrededor de las opciones uno y dos), al obtener la desviación estándar más alta (1.34), alrededor de la media de 1.94 . 
Tabla 2. Medias, desviaciones estándar, asimetría y curtosis de los 65 ítems iniciales de

IDentifica2.0

\begin{tabular}{|c|c|c|c|c|c|c|c|c|c|}
\hline Ítems & $M$ & $D E$ & Asimetría & Curtosis & Ítems & $\mathrm{M}$ & $\mathrm{DE}$ & Asimetría & Curtosis \\
\hline CID1 & 2.74 & 1.10 & -0.81 & 0.16 & PYS34 & 2.81 & 1.08 & -0.77 & 0.11 \\
\hline CID2 & 1.78 & 1.32 & 0.25 & -1.01 & PYS35 & 3.19 & 1.12 & -1.38 & 0.97 \\
\hline CID3 & 2.19 & 1.13 & -0.14 & -0.98 & PYS36 & 3.52 & 0.80 & -2.11 & 5.29 \\
\hline CID4 & 2.70 & 1.08 & -0.50 & -0.53 & PYS37 & 3.61 & 0.66 & -2.19 & 6.50 \\
\hline CID5 & 2.05 & 1.22 & 0.12 & -0.94 & PYS38 & 3.45 & 0.78 & -1.70 & 3.46 \\
\hline CID6 & 2.69 & 1.15 & -0.71 & -0.40 & PYS39 & 3.30 & 1.03 & -1.73 & 2.72 \\
\hline CID7 & 2.12 & 1.06 & 0.04 & -0.48 & RED40 & 3.38 & 0.84 & -1.38 & 1.84 \\
\hline CID8 & 2.90 & 1.12 & -0.85 & -0.06 & RED41 & 3.18 & 0.99 & -1.21 & 1.22 \\
\hline CID9 & 1.94 & 1.34 & 0.01 & -1.22 & RED42 & 3.02 & 0.93 & -0.84 & 0.55 \\
\hline CID10 & 2.04 & 1.20 & -0.08 & -0.92 & RED43 & 2.66 & 0.99 & -0.39 & -0.38 \\
\hline CID11 & 1.55 & 1.25 & 0.50 & -0.70 & RED44 & 2.63 & 0.97 & -0.59 & 0.06 \\
\hline CID12 & 2.66 & 1.00 & -0.45 & -0.23 & RED45 & 1.91 & 1.08 & 0.08 & -0.47 \\
\hline CID13 & 2.85 & 1.07 & -0.58 & -0.35 & RED46 & 1.83 & 1.19 & 0.02 & -0.77 \\
\hline VIS14 & 3.63 & 0.69 & -2.53 & 8.11 & RED47 & 3.20 & 0.81 & -1.20 & 2.37 \\
\hline VIS15 & 2.08 & 1.24 & -0.11 & -0.92 & RED48 & 2.91 & 1.02 & -0.92 & 0.61 \\
\hline VIS16 & 2.74 & 1.02 & -0.53 & -0.22 & RED49 & 1.70 & 1.27 & 0.32 & -0.84 \\
\hline VIS17 & 2.20 & 1.16 & -0.09 & -1.00 & RED50 & 2.67 & 1.16 & -0.62 & -0.51 \\
\hline VIS18 & 3.02 & 0.97 & -1.03 & 0.91 & RED51 & 2.19 & 1.24 & -0.07 & -1.08 \\
\hline VIS19 & 3.26 & 0.79 & -1.37 & 2.97 & RED52 & 3.00 & 1.09 & -1.21 & 0.96 \\
\hline VIS20 & 3.34 & 0.72 & -1.44 & 3.92 & EID53 & 2.86 & 1.08 & -0.59 & -0.47 \\
\hline VIS21 & 2.54 & 1.19 & -0.44 & -0.65 & EID54 & 1.53 & 1.20 & 0.50 & -0.65 \\
\hline VIS22 & 2.71 & 0.98 & -0.71 & 0.41 & EID55 & 1.85 & 1.32 & 0.14 & -1.07 \\
\hline VIS23 & 2.99 & 1.03 & -1.32 & 1.69 & EID56 & 1.88 & 1.32 & -0.08 & -1.11 \\
\hline VIS24 & 2.14 & 1.19 & -0.25 & -0.70 & EID57 & 1.85 & 1.23 & 0.14 & -0.85 \\
\hline VIS25 & 1.76 & 1.08 & 0.10 & -0.31 & EID58 & 2.05 & 1.30 & -0.09 & -0.85 \\
\hline VIS26 & 2.84 & 1.01 & -0.83 & 0.28 & EID59 & 1.78 & 1.23 & 0.18 & -0.84 \\
\hline PYS27 & 3.22 & 0.96 & -1.39 & 1.69 & EID60 & 1.48 & 1.31 & 0.60 & -0.66 \\
\hline PYS28 & 3.11 & 0.88 & -1.17 & 1.67 & EID61 & 2.21 & 1.27 & -0.16 & -0.94 \\
\hline
\end{tabular}




\begin{tabular}{|l|c|c|c|c|c|c|c|c|c|}
\hline PYS29 & 2.61 & 1.25 & -0.47 & -0.93 & EID62 & 2.43 & 1.09 & -0.13 & -0.69 \\
\hline PYS30 & 2.82 & 1.22 & -0.91 & -0.18 & EID63 & 2.79 & 1.17 & -0.74 & -0.28 \\
\hline PYS31 & 3.17 & 1.12 & -1.32 & 0.89 & EID64 & 3.00 & 1.14 & -1.03 & 0.29 \\
\hline PYS32 & 2.58 & 1.15 & -0.47 & -0.46 & EID65 & 2.99 & 1.16 & -0.93 & -0.12 \\
\hline PYS33 & 2.99 & 1.03 & -1.00 & 0.70 & & & & & \\
\hline
\end{tabular}

CID = Construcción de la identidad digital; VIS = Visibilidad; PYS = Privacidad y seguridad en Internet;

RED = Reputación digital; EID = Efectos de la identidad digital en el ámbito personal.

Fuente: Elaboración propia

\section{Análisis de fiabilidad}

Se determinó la confiabilidad de los ítems mediante el estadístico alfa de Cronbach. El criterio de inclusión establecido fue considerando aquellos ítems con correlaciones superiores a 0.30 respecto al total de la escala (DeVellis, 2012). Como resultado del análisis de consistencia interna, se decidió eliminar 18 ítems del cuestionario que no cumplieron con el criterio de inclusión. Como se aprecia en la tabla 3, siete ítems corresponden a la escala de Construcción de la identidad digital (CID1, CID2, CID3, CID8, CID9, CID10 Y CID11), dos a la escala de Visibilidad (VIS14 Y VIS25), dos a Privacidad y seguridad en Internet (PYS28 Y PYS39), cinco de Reputación digital (RED40, RED41, RED42, RED48 Y RED49) y dos de Efectos de la identidad digital (EID62 Y EID65).

Tabla 3. Alfa de Cronbach de la escala IDentifica2.0 con los ítems originales y alfa después de eliminar ítems

\begin{tabular}{|l|c|c|c|c|}
\hline Subescala & $\begin{array}{c}\text { Alfa } \\
\text { inicial }\end{array}$ & $\begin{array}{c}\text { Número de } \\
\text { ítems originales }\end{array}$ & $\begin{array}{c}\text { Alfa eliminando } \\
\text { los ítems }\end{array}$ & $\begin{array}{c}\text { Número de } \\
\text { ítems finales }\end{array}$ \\
\hline CID & 0.66 & 13 & 0.77 & 6 \\
\hline VIS & 0.81 & 13 & 0.81 & 11 \\
\hline PYS & 0.81 & 13 & 0.82 & 11 \\
\hline RED & 0.77 & 13 & 0.76 & 11 \\
\hline EDID & 0.82 & 13 & 0.84 & 8 \\
\hline
\end{tabular}

CID = Construcción de la identidad digital; VIS = Visibilidad; PYS = Privacidad y seguridad en

Internet; RED = Reputación digital; EDID = Efectos de la identidad digital en el ámbito personal. 


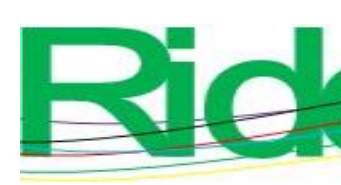

Revista Iberoamericana para la Investigación y el Desarrollo Educativo ISSN 2007 - 7467

la identidad digital en el ámbito personal (EID56). En total, los ítems finales fueron 38 repartidos en 11 factores, tal como se muestra en la tabla 4.

Tabla 4. Resultados del AFE de las subescalas que componen el cuestionario IDentifica2.0

\begin{tabular}{|l|c|c|c|c|c|c|c|}
\hline \multicolumn{1}{|c|}{ Escala } & KMO & $\chi^{2}$ & $\mathrm{gl}$ & $\chi^{2} / \mathrm{gl}$ & $\%$ de var & factores & ítems \\
\hline CID & 0.67 & 288.44 & 10 & 28.84 & $77.4 \%$ & 2 & 5 \\
\hline VIS & 0.73 & 586.93 & 21 & 27.95 & $61.9 \%$ & 2 & 7 \\
\hline PYS & 0.78 & 622.42 & 55 & 11.32 & $63.3 \%$ & 3 & 11 \\
\hline RED & 0.65 & 331.60 & 10 & 33.16 & $68.6 \%$ & 2 & 5 \\
\hline EDID & 0.81 & 881.00 & 45 & 19.58 & $50.9 \%$ & 2 & 10 \\
\hline
\end{tabular}

Nota. CID = Construcción de la identidad digital; VIS = Visibilidad; PYS = Privacidad y seguridad en Internet; RED = Reputación digital; EDID = Efectos de la identidad digital en el ámbito personal.

Fuente: Elaboración propia

\section{Análisis factorial confirmatorio (AFC)}

A fin de corroborar la asociación de factores obtenida en el AFE, se llevó a cabo el AFC tomando como criterio base la teoría del instrumento y los análisis factoriales congruentes con el diseño de las subescalas. Además, se consideró el mínimo de tres ítems por factor. Para tal fin, se empleó el método de estimación de máxima verosimilitud para determinar la bondad de ajuste empírica del modelo.

Como resultado del AFC, se eliminaron los ítems que no se asociaron con los factores del modelo (Byrne, 2010; Cea, 2004). De esta forma, se obtuvieron los modelos de medida por cada escala que cumplieron con los índices de bondad de ajuste, a fin de confirmar la sustentabilidad empírica del modelo. Los índices considerados fueron: el índice ji al cuadrado sobre grados de libertad o relativa $\left(\chi^{2} / \mathrm{gl}\right)$, la raíz cuadrada de residual estandarizada (SRMR), el índice de bondad de ajuste ajustado (AGFI), el índice de ajuste comparativo (CFI) y, finalmente, el error de la raíz cuadrada de la media de aproximación (RMSEA). Estos índices se consideran aceptables si sus valores superan los criterios de ajuste establecidos, que son $\chi^{2} / \mathrm{gl}>1$; CFI y AGFI > 0.95; SRMR < 0.08 y RMSEA < 0.06 (Brown, 2015; Hooper, Coughlan y Mullen, 2008; Hu y Bentler, 1999;). 
Figura 1. Modelos de medida de la escala IDentifica2.0 (subescalas CID, VIS, PYS, RED y EDID)

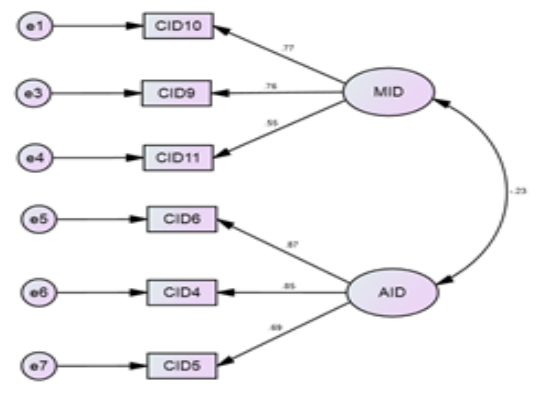

Modelo de medida para la subescala CID

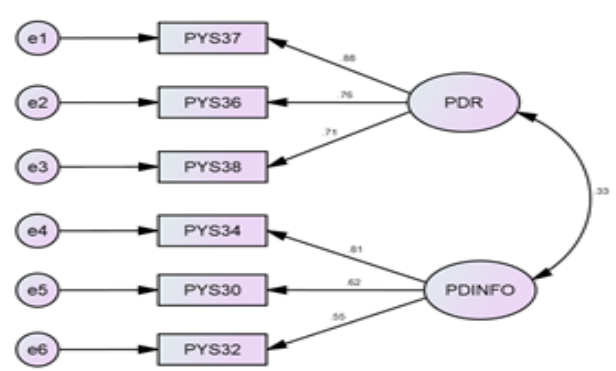

Modelo de medida para la subescala PYS

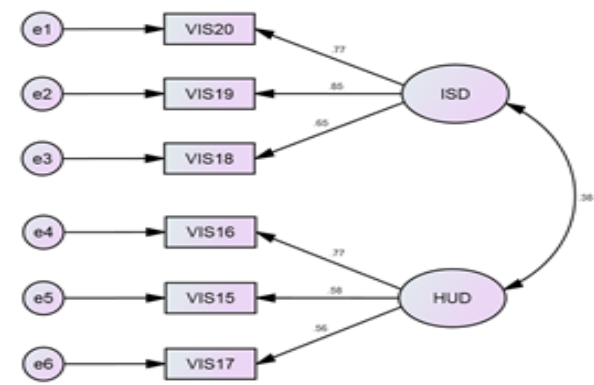

Modelo de medida para la subescala VIS

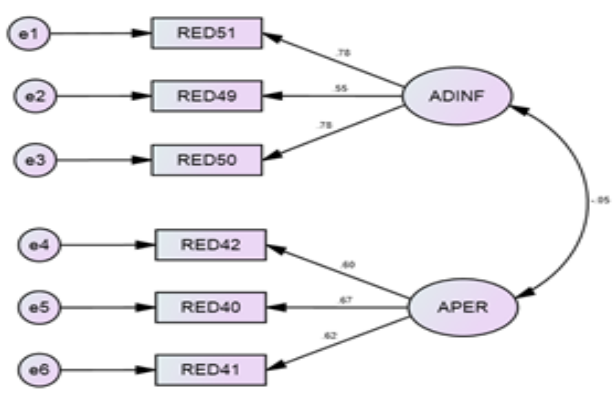

Modelo de medida para la subescala RED

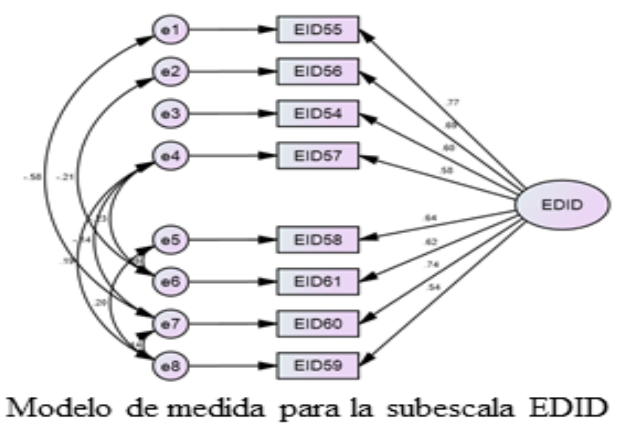

Fuente: Elaboración propia

\section{Discusión}

El proceso de validez y confiabilidad relatado da cuenta de los hallazgos encontrados acerca de la escala IDentifica2.0, con un alfa de Cronbach de 0.80 y un índice KMO de 0.728 en las cinco subescalas que comprende, permite afirmar la comprobación de la hipótesis planteada, por lo que se puede emplear en estudiantes universitarios para medir la gestión de la identidad digital. Destaca como fortaleza la integración de las subescalas que la conforman; algunos instrumentos cuantitativos que miden la gestión de la identidad digital posicionan a la identidad digital como 


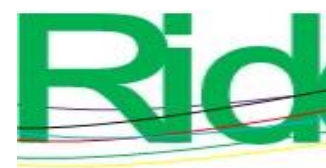

Revista Iberoamericana para la Investigación y el Desarrollo Educativo ISSN 2007 - 7467

bondad de ajuste, por lo que se recomienda manejarlo como Efectos de la identidad digital, sin hacer distinciones entre la dirección del efecto.

En cuanto a la confiabilidad, el alfa de Cronbach global de las cinco escalas fue alto (0.80), lo que indica que, en su conjunto, las subescalas miden el constructo para el cual fueron creadas, por lo que la escala IDentifica2.0 permite la medición de la gestión de la identidad digital. Tras los análisis aquí presentados, la escala queda conformada por cinco factores y 32 ítems.

De manera general, en las cinco subescalas que conforman el instrumento se encontraron varias oportunidades de mejora para obtener la evidencia empírica necesaria para sostener los constructos que se buscan identificar. Con lo anterior, se comprueba la hipótesis inicial, y se concluye que la escala IDentifica2.0 posee niveles aceptables de confiabilidad para medir la gestión de la identidad digital en estudiantes universitarios. El número de ítems adecuados para cada uno de los cuatro constructos es apenas de tres (seis por cada dimensión), excepto el indicador EDID, que quedó de seis, por lo que se sugiere pilotear nuevos indicadores que mejoren la identificación de un atributo o variable.

\section{Futuras líneas de investigación}

A partir de los resultados aquí descritos, pueden generarse futuras líneas de investigación como el diseño de instrumentos cuantitativos que evalúen conocimientos o habilidades sobre la gestión de la identidad digital, así como estudios con grupos poblacionales distintos como estudiantes de bachillerato o posgrado. Se identifica como área de oportunidad la medición en profesores de diversos niveles educativos, al igual que otras profesiones, lo que permitirá valorar estos constructos presentados. 


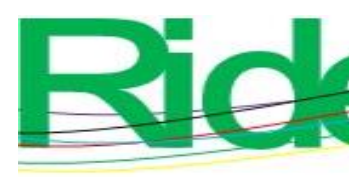

Revista Iberoamericana para la Investigación y el Desarrollo Educativo ISSN $2007-7467$

\section{Referencias}

Aced, C., Arqués, N., Benítez, M., Llodrà, B. y Sanagustín, E. (2009). Visibilidad: cómo gestionar la reputación en Internet. España: Gestión 2000.

Adriaanse, L. and Rensleigh, C. (2017). E-visibility of environmental science researchers at the University of South Africa. South African Journal of Libraries and Information Science, 83(2), 30-41. Retrieved from https://doi.org/10.7553/83-2-1636.

Aguilar, D. E., y Said, E. (2010). Identidad y subjetividad en las redes sociales virtuales: caso de Facebook. Zona Próxima, (12), 190-207. Recuperado de https://bit.ly/3ejELV6.

Ahlquist, J. (2016). The Digital Identity of Student Affairs Professionals. New Directions for Student Services, 2016(155), 29-46. Retrieved from https://doi.org/10.1002/ss.20181.

Ainsa, T. (2016). College students' digital identity: perceptions towards employment and career. College Student Journal, 50(1), 47-53. Retrieved from https://bit.ly/2JXlr1Z.

Baladán, F. y Hernández, J. (2016). Nuevas manifestaciones del relacionamiento laboral: ERecruiting, reputación digital, trabajo 3.0 y relaciones laborales en la economía colaborativa. Ponencia presentada en el XVI Simposio Argentino de Informática y Derecho (SID 2016)-JAIIO 45. Buenos Aires, del 5 al 9 de septiembre de 2016. Recuperado de https://bit.ly/3caBQMI.

Beck, E. N. (2015). The invisible digital identity: Assemblages in digital networks. Computers and Composition, 35, 125-140. $\quad$ Retrieved from https://doi.org/10.1016/j.compcom.2015.01.005.

Bjerede, M. (2015). Managing Student Identities in the Digital Era. THE Journal (Technological Horizons in Education), 42(6), 6. Retrieved from https://bit.ly/3c7ul9g.

Bozkurt, A. and Tu, C. H. (2016). Digital identity formation: Socially being real and present on digital networks. Educational Media International, 53(3), 153-167. Retrieved from https://doi.org/10.1080/09523987.2016.1236885.

Brown, T. (2015). Confirmatory Factor Analysis for Applied Research (2 ${ }^{\text {nd }}$ ed.). New York, United States: The Guilford Press.

Byrne, B. (2010). Structural Equation Modeling with AMOS. (2 ${ }^{\text {nd }}$ ed.). New York, United States: Routledge Taylor \& Francis Group.

Castañeda, L. y Camacho, M. (2012). Desvelando nuestra identidad digital. El Profesional de la Información, 21(4), 354-360. Recuperado de https://doi.org/10.3145/epi.2012.jul.04. 


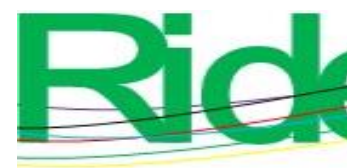

Revista Iberoamericana para la
Investigación y el Desarrollo Educativo
ISSN $2007-7467$

Castillejos, B., Torres, C. A., y Lagunes, A. (2016). La seguridad en las competencias digitales de los millennials. Apertura, 8(2), 54-69. Recuperado de https://doi.org/10.32870/ap.v8n2.914.

Cea, M. (2004). Análisis multivariable. Teoría y práctica en la investigación social. Madrid, España: Síntesis.

D’Antona, R., Kevorkian, M. and Russom, A. (2010). Sexting, Texting, Cyberbullying and Keeping Youth Safe Online. Journal of Social Sciences, 6(4), 523-528. Retrieved from https://doi.org/10.3844/jssp.2010.523.528.

Dalton, J. C. and Crosby, P. C. (2013). Digital identity: How social media are influencing student learning and development in college. Journal of College and Character, 14(1), 1-4. Retrieved from https://doi.org/10.1515/jcc-2013-0001.

Dans, I. (2015). Identidad digital de los adolescentes: la narrativa del yo. Revista de Estudios e Investigación en Psicología y Educación, (13), 1-4. Recuperado de https://doi.org/10.17979/reipe.2015.0.13.145.

Davis, K. (2013). Young people's digital lives: The impact of interpersonal relationships and digital media use on adolescents' sense of identity. Computers in Human Behavior, 29(6), 22812293. Retrieved from https://doi.org/10.1016/j.chb.2013.05.022.

de Frutos, B. y Vázquez, T. (2014). Adolescentes y jóvenes en el entorno digital: Análisis de su discurso sobre usos, percepción de riesgo y mecanismos de protección, doxa.comunicación, $\quad$ (15), 57-79. Recuperado de https://doi.org/10.31921/doxacom.n15a3.

DeVellis, R. (2012). Scale Development. Theory and Applications. New York, United States: SAGE.

Dutot, V. and Castellano, S. (2015). Designing a measurement scale for e-reputation. Corporate Reputation Review, 18(4), 294-313. Retrieved from https://doi.org/10.1057/crr.2015.15.

Fernández, C. S. (2014). La vida privada en la sociedad digital. La exposición pública de los jóvenes en internet. Aposta. Revista de Ciencias Sociales, (61), 1-32. Recuperado de https://bit.ly/3b7jVGD.

Ferrari, A. (2013). DIGCOMP: A Framework for Developing and Understanding Digital Competence in Europe. Sevilla, España: Joint Research Centre of the European Commission. 


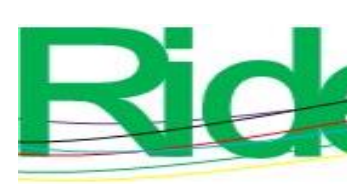

Revista Iberoamericana para la Investigación y el Desarrollo Educativo ISSN 2007 - 7467

Gabrielidis, A. L. (2015). Régimen de visibilidad y vigilancia en la era de la identidad digital. Revista Teknokultura, 12(3), 473-499. Recuperado de https://doi.org/10.5209/rev_tk.2015.v12.n3.50385.

Galera, M., Hurtado, M. y Fernández, C. (2014). Las redes sociales en la cultura digital: percepción, participación, movilización. Revista de la Asociación Española de Investigación de la Comunicación, 1(1), 12-18. Recuperado de https://bit.ly/2V56Bx1.

Gamero, R. (2009). La configuración de la identidad digital. Nota Enterie, (131), 1-6. Recuperado de https://cursa.ihmc.us/rid=1H8FQCJ5D-R3NH1347X/acerca_de_la_identidad_digital.pdf .

García, M. D., del Olmo, J. y del Hoyo, M. (2017). Jóvenes, privacidad y dependencia en las redes sociales. Ponencia presentada en el II Congreso Internacional Move net sobre Movimientos Sociales y TIC. Sevilla, del 25 al 27 de octubre de 2017. Recuperado de https://hdl.handle.net/11441/70629.

Georges, F. (2011). L'identité numérique sous emprise culturelle. Les Cahiers du numérique, 7(1), 31-48. Récupéré de https://bit.ly/2V2TM65.

Giones, V. A. y Serrat, B. M. (2010). La gestión de la identidad digital: una nueva habilidad informacional y digital. BiD: Textos Universitaris de Biblioteconomia i Documentació, (24). Recuperado de https://bit.ly/2XtRX3W.

Griffin, M. M. and Steinbrecher, T. D. (2013). Large-Scale Datasets in Special Education Research. In Urbano, R. (ed.), International Review of Research in Developmental Disabilities (pp. 155-183). Waltham, United States: Elsevier. Retrieved from https://doi.org/10.1016/b978-0-12-407760-7.00004-9.

Harrell, D. F. and Lim, C. U. (2017). Reimagining the avatar dream: Modeling social identity in digital media. Communications of the ACM, 60(7), 50-61. Retrieved from https://doi.org/10.1145/3098342.

Herrera, M., Casas, J. A., Romera, E. M., Ortega, R. and del Rey, R. (2017). Validation of the European Cyberbullying Intervention Project Questionnaire for Colombian Adolescents. Cyberpsychology, Behavior, and Social Networking, 20(2), 117-125. Retrieved from https://doi.org/10.1089/cyber.2016.0414.

Hooper, D., Coughlan, J. and Mullen, M. (2008). Structural Equation Modelling: Guidelines for Determining Model Fit. Electronic Journal of Business Research Methods, 6(1), 53-60. Retrieved from https://bit.ly/2VrLxzJ. 


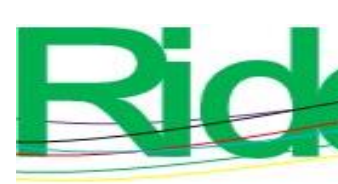

Revista Iberoamericana para la Investigación y el Desarrollo Educativo ISSN 2007 - 7467

Hu, L. T. and Bentler, P. M. (1999). Cutoff criteria for fit indexes in covariance structure analysis: Conventional criteria versus new alternatives. Structural Equation Modeling: A Multidisciplinary Journal, 6(1), 1-55. Retrieved from https://doi.org/10.1080/10705519909540118.

Kelly, S., Christen, S. and Snyder, L. G. (2013). An Analysis of Effective Online Reputation Management: A Critical Thinking Social Media Activity. The Journal of Research in Business Education, 55(1), 24-35. Retrieved from https://bit.ly/2ycxp5e.

Kim, M. and Choi, D. (2018). Development of Youth Digital Citizenship Scale and Implication for Educational Setting. Journal of Educational Technology \& Society, 21(1), 155-171. Retrieved from https://www.jstor.org/stable/26273877?seq=1\#metadata_info_tab_contents.

Kline, R. (2016). Principles and Practice of Structural Equation Modeling (4 ${ }^{\text {th }}$ ed.). New York, United States: The Guilford Press.

Lara, T. (2009). El papel de la universidad en la construcción de su identidad digital. Revista de la Universidad y Sociedad del Conocimiento, 6(1), 15-21. Recuperado de http://dx.doi.org/10.7238/rusc.v6i1.25.

Mannerström, R., Hietajärvi, L., Muotka, J. and Salmela-Aro, K. (2018). Identity profiles and digital engagement among Finnish high school students. Cyberpsychology, 12(1). Retrieved from https://doi.org/10.5817/cp2018-1-2.

Meraz, A. I. (2018). Empresa y privacidad: el cuidado de la información y los datos personales en medios digitales. Revista IUS, 12(41), 293-310. Recuperado de https://doi.org/10.35487/rius.v12i41.2018.313.

Moreno, B. T., Gajardo, L. C., y Parra, O. E. (2016). Privacidad: cómo se entiende y se gestiona en Facebook. Estudio de caso de jóvenes chilenos. Revista latina de comunicación social, (71), 715-729. Recuperado de https://doi.org/10.4185/rlcs-2016-1117.

Nicholas, D. (2017). New ways of building, showcasing, and measuring scholarly reputation in the digital age. Information Services \& Use, 37(1), 1-5. Retrieved from https://doi.org/10.3233/isu-160792.

Núñez, S. y Manolakis, L. (2016). Reputación digital del alumno universitario: mucho más que tener "un millón de amigos" en Facebook. En Iriondo, W., Bras, I., Mussi, P. y Gamboa, M. C. (eds.), Utilizando tecnologías en la educación para fortalecer la práctica docente 

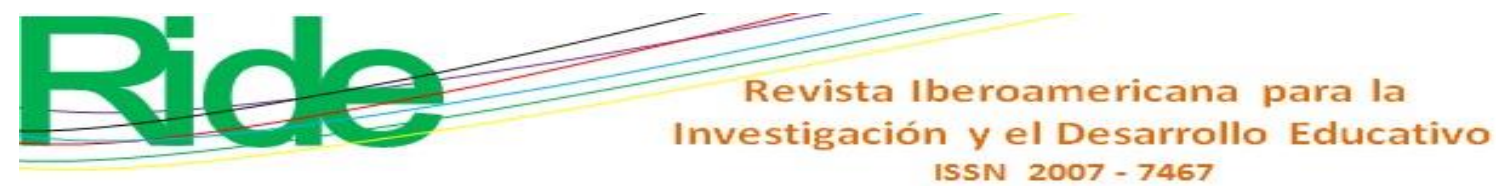

en América Latina. Revisiones teóricas-Experiencias prácticas (pp. 334-342). Recuperado de https://bit.ly/3cayg5l.

Ortega R., del Rey, R. y Casas, J. A. (2016). Evaluar el bullying y el cyberbullying: validación española del EBIP-Q y del ECIP-Q. Psicología Educativa, 22(1), 71-79. Recuperado de https://doi.org/10.1016/j.pse.2016.01.004.

Peachey, A. and Childs, M. (2011). Virtual Worlds and Identity. In Peachey, A. and Childs, M. (eds.), Reinventing Ourselves: Contemporary Concepts of Identity in Virtual Worlds (pp. 1-12). London, England: Springer. Retrieved from https://doi.org/10.1007/978-0-85729361-9_1.

Perea, F. (2010). L'identité numérique: de la cité à l'écran. Quelques aspects de la représentation de soi dans l'espace numérique. Les Enjeux de l'information et de la communication, 2010(1), 144-159. Récupéré de https://doi.org/10.3917/enic.010.0800.

Pérez, S. M. (2012). Identidad digital. Telos, (91). Recuperado de https://bit.ly/2yczgqI.

Portillo, F. J. (2016). Planos de realidad, identidad virtual y discurso en las redes sociales. Logos, 26(1), 51-63. Recuperado de https://doi.org/10.15443/rl2604.

Ramos, I., López, C. y Torrecillas, T. (2018). Percepción de riesgo online en jóvenes y su efecto en el comportamiento digital. Comunicar, 26(56), 71-79. Recuperado de https://doi.org/10.3916/C56-2018-07.

Rodríguez, L. y Magdalena, J. R. (2016). Perspectiva de los jóvenes sobre seguridad y privacidad en las redes sociales. Icono14, 14(1), 24-49. Recuperado de https://doi.org/10.7195/ri14.v14i1.885.

Sabater, C. y López, L. (2015). Factores de riesgo en el ciberbullying. Frecuencia y exposición de los datos personales en Internet. International Journal of Sociology of Education, 4(1), 125. Recuperado de http://dx.doi.org/10.4471/rise.2015.01.

Saiz, C., Ronco, M. M. y Echegaray, L. (2017). Jóvenes, salud e Internet. Percepción, actitud y motivaciones de los jóvenes ante la información de salud. Revista Latina de Comunicación Social, (72), 1317-1340. Recuperado de https://doi.org/10.4185/rlcs-20171221.

Seker, S. E. and Eryarsoy, E. (2015). Generating digital reputation index: A case study. ProcediaSocial and Behavioral Sciences, 195, 1074-1080. Retrieved from https://doi.org/10.1016/j.sbspro.2015.06.151. 


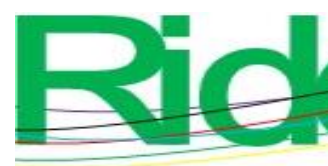
Revista Iberoamericana para la
Investigación y el Desarrollo Educativo
ISSN $2007-7467$

So, K. K. F., Wu, L., Xiong, L. and King, C. (2018). Brand Management in the Era of Social Media: Social Visibility of Consumption and Customer Brand Identification. Journal of Travel Research, 57(6), 727-742. Retrieved from https://doi.org/10.1177/0047287517718354.

Telci, E. E. and Kantur, D. (2014). University Reputation: Scale Development and Validation. Bogazici Journal: Review of Social, Economic \& Administrative Studies, 28(2). Retrieved from https://doi.org/10.21773/boun.28.2.4.

Tusa, J. F. (2018). La construcción de la identidad digital y las narrativas híbridas. (Tesis doctoral). Universidad Austral, Buenos Aires. Recuperado de http://hdl.handle.net/123456789/604.

Valderrama, B. M. (2016). El devenir de la identidad digital: del yo proteico al yo identificado. PAAKAT: Revista de Tecnología y Sociedad, (11). Recuperado de https://doi.org/10.32870/pk.a6n11.282.

Van, O. W. and Steinfield, C. W. (2018). Strategic visibility in enterprise social media: Implications for network formation and boundary spanning. Journal of Management Information Systems, 35(2), 647-682. Retrieved from https://doi.org/10.1080/07421222.2018.1451961.

Vargas, A. (2016). Redes sociales, literacidad e identidad (es): el caso de Facebook. Colombian Applied Linguistics Journal, 18(1), 11-23. Recuperado de https://doi.org/10.14483/calj.v18n1.9415.

Wise, J. B. and O’Byrne, W. I. (2015). Social Scholars: Educators' Digital Identity Construction in Open, Online Learning Environments. Literacy Research: Theory, Method, and Practice, 64(1), 398-414. Retrieved from https://doi.org/10.1177/2381336915617607. 


\begin{tabular}{|c|c|}
\hline Rol de Contribución & Autor (es) \\
\hline Conceptualización & $\begin{array}{l}\text { Gustavo Antonio Huerta Patraca y Carlos Arturo Torres } \\
\text { Gastelú (igual) }\end{array}$ \\
\hline Metodología & $\begin{array}{l}\text { Gustavo Antonio Huerta Patraca y Carlos Arturo Torres } \\
\text { Gastelú (igual) }\end{array}$ \\
\hline Software & Gustavo Antonio Huerta Patraca \\
\hline Validación & $\begin{array}{l}\text { Gustavo Antonio Huerta Patraca, Carlos Arturo Torres Gastelú } \\
\text { y Agustín Lagunes Domínguez (igual) }\end{array}$ \\
\hline Análisis Formal & $\begin{array}{l}\text { Gustavo Antonio Huerta Patraca, Carlos Arturo Torres Gastelú } \\
\text { y Agustín Lagunes Domínguez (igual) }\end{array}$ \\
\hline Investigación & $\begin{array}{l}\text { Gustavo Antonio Huerta Patraca, Carlos Arturo Torres Gastelú } \\
\text { y Agustín Lagunes Domínguez (igual) }\end{array}$ \\
\hline Recursos & $\begin{array}{l}\text { Gustavo Antonio Huerta Patraca, Carlos Arturo Torres Gastelú } \\
\text { y Agustín Lagunes Domínguez (igual) }\end{array}$ \\
\hline Curación de datos & Gustavo Antonio Huerta Patraca \\
\hline $\begin{array}{l}\text { Escritura - Preparación del } \\
\text { borrador original }\end{array}$ & Gustavo Antonio Huerta Patraca \\
\hline $\begin{array}{l}\text { Escritura - Revisión y } \\
\text { edición }\end{array}$ & $\begin{array}{l}\text { Gustavo Antonio Huerta Patraca, Carlos Arturo Torres Gastelú } \\
\text { y Agustín Lagunes Domínguez (igual) }\end{array}$ \\
\hline Visualización & $\begin{array}{l}\text { Gustavo Antonio Huerta Patraca, Carlos Arturo Torres Gastelú } \\
\text { y Agustín Lagunes Domínguez (igual) }\end{array}$ \\
\hline Supervisión & Gustavo Antonio Huerta Patraca \\
\hline Administración de Proyectos & Gustavo Antonio Huerta Patraca \\
\hline Adquisición de fondos & No aplica \\
\hline
\end{tabular}

